\title{
Imaging-Based Outcome Prediction of Acute Intracerebral Hemorrhage
}

\author{
Jawed Nawabi ${ }^{1,2}$ (1) Helge Kniep ${ }^{1} \cdot$ Sarah Elsayed $^{1} \cdot$ Constanze Friedrich $^{1} \cdot$ Peter Sporns $^{1,4} \cdot$ Thilo Rusche $^{4}$. \\ Maik Böhmer ${ }^{5}$ - Andrea Morotti ${ }^{6}$ - Frieder Schlunk ${ }^{3}$. Lasse Dührsen ${ }^{7} \cdot$ Gabriel Broocks ${ }^{1} \cdot$ Gerhard Schön $^{8}$. \\ Fanny Quandt ${ }^{9}$. Götz Thomalla ${ }^{8}$. Jens Fiehler ${ }^{1}$ • Uta Hanning ${ }^{1}$
}

Received: 1 September 2020 / Revised: 3 January 2021 / Accepted: 12 January 2021 / Published online: 6 February 2021

(C) The Author(s) 2021

\begin{abstract}
We hypothesized that imaging-only-based machine learning algorithms can analyze non-enhanced CT scans of patients with acute intracerebral hemorrhage (ICH). This retrospective multicenter cohort study analyzed 520 non-enhanced CT scans and clinical data of patients with acute spontaneous ICH. Clinical outcome at hospital discharge was dichotomized into good outcome and poor outcome using different modified Rankin Scale (mRS) cut-off values. Predictive performance of a random forest machine learning approach based on filter- and texture-derived high-end image features was evaluated for differentiation of functional outcome at mRS 2,3 , and 4 . Prediction of survival (mRS $\leq 5)$ was compared to results of the ICH Score. All models were tuned, validated, and tested in a nested 5fold cross-validation approach. Receiver-operating-characteristic area under the curve (ROC AUC) of the machine learning classifier using image features only was 0.80 (95\% CI [0.77; 0.82]) for predicting $\mathrm{mRS} \leq 2,0.80$ (95\% CI $[0.78 ; 0.81])$ for $\mathrm{mRS} \leq 3$, and 0.79 $(95 \%$ CI $[0.77 ; 0.80])$ for $\mathrm{mRS} \leq 4$. Trained on survival prediction $(\mathrm{mRS} \leq 5)$, the classifier reached an AUC of 0.80 (95\% CI $[0.78$; $0.82]$ ) which was equivalent to results of the ICH Score. If combined, the integrated model showed a significantly higher AUC of 0.84 (95\% CI $[0.83 ; 0.86], P$ value $<0.05)$. Accordingly, sensitivities were significantly higher at Youden Index maximum cut-offs $(77 \%$ vs. $74 \%$ sensitivity at $76 \%$ specificity, $P$ value $<0.05$ ). Machine learning-based evaluation of quantitative high-end image features provided the same discriminatory power in predicting functional outcome as multidimensional clinical scoring systems. The integration of conventional scores and image features had synergistic effects with a statistically significant increase in AUC.
\end{abstract}

Keywords Intracerebral hemorrhage $\cdot$ Outcome prediction $\cdot$ Radiomics $\cdot$ Machine Learning

\section{Introduction}

Intracerebral hemorrhage ( $\mathrm{ICH})$ is the most severe form of stroke with a 1-month morbidity and mortality approaching

Jawed Nawabi and Helge Kniep contributed equally to this work.

Jawed Nawabi

jawed.nawabi@charite.de

1 Department of Diagnostic and Interventional Neuroradiology, University Medical Center Hamburg Eppendorf, Hamburg, Germany

2 Department of Radiology, Charité - Universitätsmedizin Berlin, Campus Mitte, Campus Mitte, Humboldt-Universität zu Berlin, Freie Universität Berlin, Berlin Institute of Health (BIH), BIH Biomedical Innovation Academy, Berlin, Germany

3 Department of Radiology, Charité School of Medicine and University Hospital Berlin, Berlin, Germany
$50 \%$ and death or severe disability exceeding $75 \%$ [1-3]. In contrast to recent advances in interventional treatments of patients with ischemic stroke, beneficial effects of medical treatment and surgical intervention on the mortality and functional

4 Department of Neuroradiology, Clinic for Radiology and Nuclear Medicine, University Hospital Basel, Basel, Switzerland

5 Department of Radiology, University Hospital Muenster, Muenster, Germany

6 Neurology Unit, ASST Valcamonica, Esine, BS, Italy

7 Department of Neurosurgery, University Medical Center Hamburg-Eppendorf, Hamburg, Germany

8 Institute of Medical Biometry and Epidemiology, University Medical Center Hamburg-Eppendorf, Hamburg, Germany

9 Department of Neurology, University Medical Center Hamburg-Eppendorf, Hamburg, Germany 
outcome of ICH patients were not observed in recent trials [4, 5]. Accurate stratification of ICH prognosis is highly desired regardless of the therapeutic options that are available and remains a clinical research priority [6]. Therefore, several prognostic tools have been proposed for the prediction of mortality and functional outcome in spontaneous ICH [7]. Though potentially useful for ascertaining prognosis and facilitating communication between clinicians, numerous methodological and reporting deficiencies are reported for a majority of these tools [7]. There is growing interest in augmented diagnostic and prognostic vision with machine learning (ML) in the medical field due to the wide range of applications of these algorithms and the increasing availability of computational power. ML is a type of artificial intelligence that learns patterns and rules from given information [8]. Recent studies applied ML to severity and outcome prediction models for neurological disorders such as ischemic stroke [8], aneurysmal subarachnoid hemorrhage [9], and traumatic brain injury [10]. However, ML approaches in the field of ICH were mainly focused on prompt diagnosis and automated volume quantification $[11,12]$ with lacking algorithms for the prediction of clinical outcome. As of late, Wang et al. have been among the first to develop an outcome prediction model based on ML by incorporating initial clinical presentations, laboratory data, and imaging findings [13]. Imaging findings were limited to ICH volume and location, presence of intraventricular hemorrhage, ventricle compression, and midline structure shift [13]. Further integration of quantitative imaging characteristics may hold additional prognostic value [9]. In the past, specific CT markers and histogram-based analyses of ICH heterogeneity have been linked to poor clinical outcome and reinforce this notion [14-16]. The goal of this study was twofold: First, we hypothesized that quantitative radiomic filter- and texturederived high-end image features extracted from nonenhanced computed tomography (NECT) brain scans can be used to predict clinical outcome of ICH patients. To test and evaluate this hypothesis, we employed a radiomics-based ML approach on NECT brain scans of patients presenting with acute primary ICH [17]. Secondly, we hypothesized that the diagnostic power of the presented algorithm using high-end image features is equal to the ICH Score serving as the most widely utilized prognostic model for predicting mortality [18].

\section{Materials and Methods}

\section{Study Population}

We retrospectively analyzed the database of three university hospitals (University Medical Center Hamburg-Eppendorf, Charité University Medical Center Berlin, University Medical Center Münster) with a high-volume tertiary stroke center, for patients with ICH aged $\geq 18$ years between January
2010 and April 2019. Inclusion criteria were defined as follows: Spontaneous ICH confirmed on NECT on admission. Patients were excluded if they had a secondary ICH from head trauma, hemorrhagic transformation of ischemic infarction, brain tumor, cerebral aneurysm, or vascular malformation. Baseline patient characteristics were retrieved from medical records, including Glasgow Coma Scale (GCS) at admission and modified Rankin Scale (mRS) at discharge. Additionally, we obtained vascular risk factors, blood pressure parameters, antiplatelet and oral anticoagulation (OAC) medication, and follow-up procedures, such as craniectomy or intraventricular drainage placement from patients' clinical records and followup CT. A binary clinical outcome was defined based on modified Rankin Scale (mRS) on discharge with $\leq 3$ as good outcome and $\mathrm{mRS}>3$ as poor outcome [19]. According to the inclusion criteria, 520 patients were included, out of which $151(29 \%)$ patients had a good outcome (mRS 0-3) and 369 (71\%) patients had a poor outcome (mRS 4-6). Details are listed for further consideration in Table 1. This multicenter retrospective study was approved by the ethics committee (Ethik-Kommission der Ärztekammer Hamburg, EthikKomission der Charité Berlin) and written informed consent was waived by the institutional review boards. All study protocols and procedures were conducted in accordance with the Declaration of Helsinki. The deidentified data and analytic code are available from the corresponding author upon reasonable request.

\section{Image Acquisitions}

The NECT scans were performed using standard clinical parameters with axial $<5 \mathrm{~mm}$ section thickness. All datasets were inspected for quality and excluded in case of severe motion artifacts. In detail, the images were acquired on the following scanners: 256 slice scanner (Philips iCT 256) with $120 \mathrm{kV}, 280-320 \mathrm{~mA},<5.0 \mathrm{~mm}$ slice reconstruction; 80 slice scanner (Toshiba Aquilion Prime) with $120 \mathrm{kV}, 280 \mathrm{~mA},<$ $5.0 \mathrm{~mm}$ slice reconstruction and $<0.5 \mathrm{~mm}$ in-plane resolution; and $2 \times 128$ slice scanner (SOMATOM Definition Flash) with $120 \mathrm{kV}, 280 \mathrm{~mA},<5.0 \mathrm{~mm}$ slice reconstruction and $<0.5 \mathrm{~mm}$ in-plane resolution.

\section{Post-procedure Evaluations}

NECT scans were obtained and stored for further evaluation. Two experienced neuroradiologists (JN and SE) assessed and documented the following imaging features on NECT scans: [1] intraventricular hemorrhage; [2] ICH location; [3] craniectomy in the follow-up NECT scans. ICH locations were classified as basal ganglia, thalamus, lobe, brain stem, pons, and cerebellum. In the following ICH, volumes were segmented semi-automatically on the basis of the original NECT images [20]. Regions of interest (ROIs) were 
Table 1 Baseline demographic, clinical, and radiological characteristics of study cohort

\begin{tabular}{|c|c|c|c|c|}
\hline Baseline characteristics & $\begin{array}{l}\text { All } \\
(n=520)\end{array}$ & $\begin{array}{l}\mathrm{mRS} 0-3 \\
(n=151)\end{array}$ & $\begin{array}{l}\mathrm{mRS} 4-6 \\
(n=369)\end{array}$ & $P$ value \\
\hline \multicolumn{5}{|l|}{ Clinical parameters } \\
\hline Age [years], median (IQR) & $73(59 ; 79)$ & $70(57 ; 78)$ & $73(60 ; 80)$ & 0.85 \\
\hline Female, $n(\%)$ & $234(45.0)$ & $67(44.4)$ & $167(45.3)$ & 0.85 \\
\hline Hypertension, $n(\%)$ & $359(69.2)$ & $99(65.6)$ & $260(70.7)$ & 0.25 \\
\hline Diabetes mellitus, $n(\%)$ & $73(14.0)$ & $23(15.2)$ & $50(13.7)$ & 0.62 \\
\hline Antiplatelet medication, $n(\%)$ & $104(20)$ & $33(21.9)$ & $71(19.2)$ & 0.50 \\
\hline Anticoagulant medication, $n(\%)$ & $113(21.7)$ & $34(22.5)$ & $79(21.4)$ & 0.78 \\
\hline $\begin{array}{l}\text { Systolic blood pressure }[\mathrm{mm} \mathrm{Hg}] \\
\text { median }(\mathrm{IQR})\end{array}$ & $162(138 ; 193.75)$ & $162(145 ; 185)$ & $160(135 ; 197.5)$ & 0.75 \\
\hline $\begin{array}{l}\text { Time from symptom onset to } \\
\text { CT [days], median (IQR) }\end{array}$ & $0.19(0.76 ; 0.52)$ & $0.21(0.09 ; 0.59)$ & $0.19(0.07 ; 0.52)$ & 0.92 \\
\hline $\begin{array}{l}\text { Time from CT to discharge [days], } \\
\text { median (IQR) }\end{array}$ & $14(7 ; 22)$ & $14(6.5 ; 18.5)$ & $15.5(6.75 ; 23.5)$ & 0.13 \\
\hline \multicolumn{5}{|l|}{ Clinical scores } \\
\hline GCS Score, median (IQR) & $11(5 ; 14)$ & $14(12 ; 15)$ & $9(3 ; 13)$ & $<0.001$ \\
\hline ICH Score, median (IQR) & $2(1 ; 3)$ & $1(0 ; 2)$ & $3(2 ; 4)$ & $<0.001$ \\
\hline \multicolumn{5}{|l|}{ CT parameters } \\
\hline \multicolumn{5}{|l|}{ Bleeding location, $n(\%)$} \\
\hline - Lobar & $238(45.8)$ & $76(50.3)$ & $162(43.9)$ & 0.18 \\
\hline - Basal ganglia & $198(38.1)$ & $54(35.8)$ & $144(39.0)$ & 0.49 \\
\hline - Thalamus & $18(3.5)$ & $3(2.0)$ & $15(4.1)$ & 0.24 \\
\hline - Brainstem and pons & $23(4.4)$ & $5(3.3)$ & $18(4.9)$ & 0.43 \\
\hline - Cerebellar & $43(8.3)$ & $13(8.6)$ & $30(8.1)$ & 0.86 \\
\hline Intraventricular hemorrhage, $n(\%)$ & $267(51.3)$ & $50(33.1)$ & $217(59)$ & $<0.001$ \\
\hline $\mathrm{ICH}$ volume $[\mathrm{mL}]$, median (IQR) & $25.1(9.7 ; 60.3)$ & $11.5(3.6 ; 24.7)$ & $35.5(14.9 ; 73.2)$ & $<0.001$ \\
\hline \multicolumn{5}{|l|}{ Surgical procedures } \\
\hline Craniectomy, median (IQR) & $117(22.5)$ & $16(10.6)$ & $101(27.4)$ & $<0.001$ \\
\hline
\end{tabular}

Comparison of baseline demographic, clinical, and radiological characteristics between ICH patients with good clinical outcome (modified Rankin Scale (mRS) 0-3) versus poor clinical outcome (mRS 4-6). ICH Score, Intracerebral Hemorrhage Score; GCS, Glasgow Coma Scale; $I Q R$, interquartile range delineated using Analyze 11.0 Software (Biomedical Imaging Resource, Mayo Clinic, Rochester, MN). Consensus ROIs were derived based on overlapping segmentations of both readers. Both readers were blinded to all clinical information and bleeding location. Discrepancies were settled by joint discussion of the 2 readers and a third reader (UH). JN and SE: 3 years clinical experience in diagnostic neuroradiology in an academic full-service hospital; UH: 8 years clinical experience in diagnostic neuroradiology; JN, SE, and UH: research with focus on clinical applications of image processing and predictive modelling.

\section{ICH Score}

ICH Scores were obtained for every patient included according to the definition of Hemphill et al. based on five independent and multidimensional predictors ( $\mathrm{ICH}$ volume, infratentorial location, GCS, age, and intraventricular extension) [18]. ICH volumes were obtained from ICH delineations. Oral anticoagulants (OAC) were not included as their addition does not increase the prognostic performance of the ICH Score [21]. As the ICH Score is a prognostic model for 30-day mortality in ICH patients (equivalent to $\mathrm{mRS} 6$ ), a binary mortality outcome was defined based on $\mathrm{mRS}$ at discharge with $\mathrm{mRS} \leq 5$ (survival) and $\mathrm{mRS}=6$ (death).

\section{Imaging-Based Outcome Prediction}

Radiomic features were defined according to the PyRadiomics Python package v2.1.0. Features were extracted from consensus ROIs and resampled to $0.5 \mathrm{~mm} \times 0.5 \mathrm{~mm} \times 2 \mathrm{~mm}$ resolution using sitk BSpline interpolators. Resampling was performed to ensure comparability of texture analysis. Extracted features comprised 252 first-order features (thereof 18 based on unfiltered images, 144 based on wavelet decompositions, 90 based on log-sigma laplacian of Gaussian filters), 902 
texture features (thereof 68 based on unfiltered images, 544 based on wavelet decompositions, 290 based on log-sigma laplacian of Gaussian filters), and 14 shape features. In total, 1218 quantitative image features were extracted from the ICH ROIs. To adjust for effects of therapeutic interventions that cannot be detected on admission NECTs, we included decompressive craniectomy as sole clinical parameter into the machine learning models.

ML-based classification was performed using random forest algorithms (Python scikit-learn environment v0.20.3 [22]). Random forest is a ML technique that utilizes multiple decision trees trained on random sub-selections of samples in order to improve stability and reduce overfitting of the algorithm [23]. Decision trees learn decision rules according to predictor values of the training data samples. With increasing depth of nodes, decision trees can represent more complex decision rules, resulting in a better fitting of the model [23, 24]. Hyperparameter tuning (total number of features, number of trees, maximum depth of the tree, minimum number of samples to split an internal node, number of features considered for splitting $\left(\mathrm{m}_{\text {try }}\right)$, minimum number of samples at leaf node, bootstrapping yes/no) was performed in a nested 5-fold crossvalidation approach for each training set using grid search algorithms. Parameters at initiation were set to scikit-learn default values.

Selection of features with highest predictive value was conducted separately for each training dataset of the 5-fold crossvalidation outer loop sample split according to Gini impurity measures [25]. Classifier models were trained and tested on each set's unique training and testing samples (outer loop) utilizing optimized hyperparameters and feature importance of the respective training data (inner loop).

\section{Integration of ICH Score and Imaging-Based Outcome Prediction}

It was shown that combinations of classification models trained on heterogeneous predictors tend to have higher synergistic effects if knowledge flows are merged at a very late stage of the data evaluation process. Therefore, probabilities for survival of the ICH Score and of the imaging-based classifier were extracted. The arithmetic average of both probabilities was then used for outcome prediction.

\section{Statistics}

Model validation and testing of all classifiers was conducted in a nested 5-fold cross-validation with independent training and validation sets in a model-external approach [26]. Accordingly, model selection and hyperparameter tuning was performed with grid search algorithms on each training data set using a second cross-validation layer. Model stability was examined through comparative analysis of 10 randomly permuted cross-validation sets.

Receiver-operating characteristic (ROC) curves were generated from prediction results of all cross-validation sets. Confidence intervals (CI) for sensitivities and specificities were bootstrapped (2000 replicates, pROC v1.15 [27] R-package). Bonferroni adjustments were applied to control for alpha error inflation.

Furthermore, the classifiers were analyzed using ROC areas under the curve (AUC), sensitivity, specificity, accuracy, Youden Index, positive predictive value, negative predictive value (ThresholdROC v2.8 R-package), and Matthews correlation coefficient (MCC) [28] metrics (psychometric v.2.2. R-package). MCC evaluates all fields of the confusion matrix and is considered a favorable measure for unbiased comparisons of binary classifiers [29]. With $T P$ : true positives, $T N$ : true negatives, $F P$ : false positives, and $F N$ : false negatives, $\mathrm{MCC}$ is defined as:

$$
M C C=\frac{T P \times T N-F P \times F N}{\sqrt{(T P+F P)(T P+F N)(T N+F P)(T N+F N)}}
$$

A flow chart of the proposed ML-based prediction of the clinical outcome is depicted in Fig. 1.

\section{Results}

Our analysis included NECT images of 520 patients with acute ICH. One hundred fifty-one patients $(29 \%)$ had a mRS of $0-3$ and $369(71 \%)$ had a mRS of 4-6. There were no statistically significant differences in clinical parameters age $(P$ value $=0.85)$, sex $(P$ value $=0.85)$, hypertension $(P$ value $=$ $0.25)$, diabetes mellitus $(P$ value $=0.62)$, antiplatelet or anticoagulant medication $(P$ value $=0.5$ and $P$ value $=0.78$, respectively), and systolic blood pressure at admission ( $P$ value $=0.75$ ). Both time from symptom onset to admission $C T$ and time from CT to hospital discharge were not statistically different $(P$ value 0.92 and $P$ value $=0.13$, respectively) However, patients with mRS 4-6 had a significantly lower GCS (GCS 9 versus GCS $14 ; P$ value $<0.001$ ), higher percentage of intraventricular hemorrhage (59\% versus $33.1 \%$; $P$ value $<0.001)$, higher ICH volumes $\left(35.2 \mathrm{~cm}^{3}\right.$ versus $8.4 \mathrm{~cm}^{3} ; P$ value $<0.001$ ), and a higher rate of supra-tentorial craniectomies $(27.4 \%$ versus $10.6 \% ; P$ value $<0.02)$. There were no significant differences in ICH locations. ICH Score was significantly higher in patients with mRS 4-6 (median 3 versus $1 ; P<0.001)$.

\section{Imaging-Based Outcome Prediction}

Machine learning-based ROC AUCs of the validation sets for predicting functional clinical outcome were $0.80(95 \%$ 


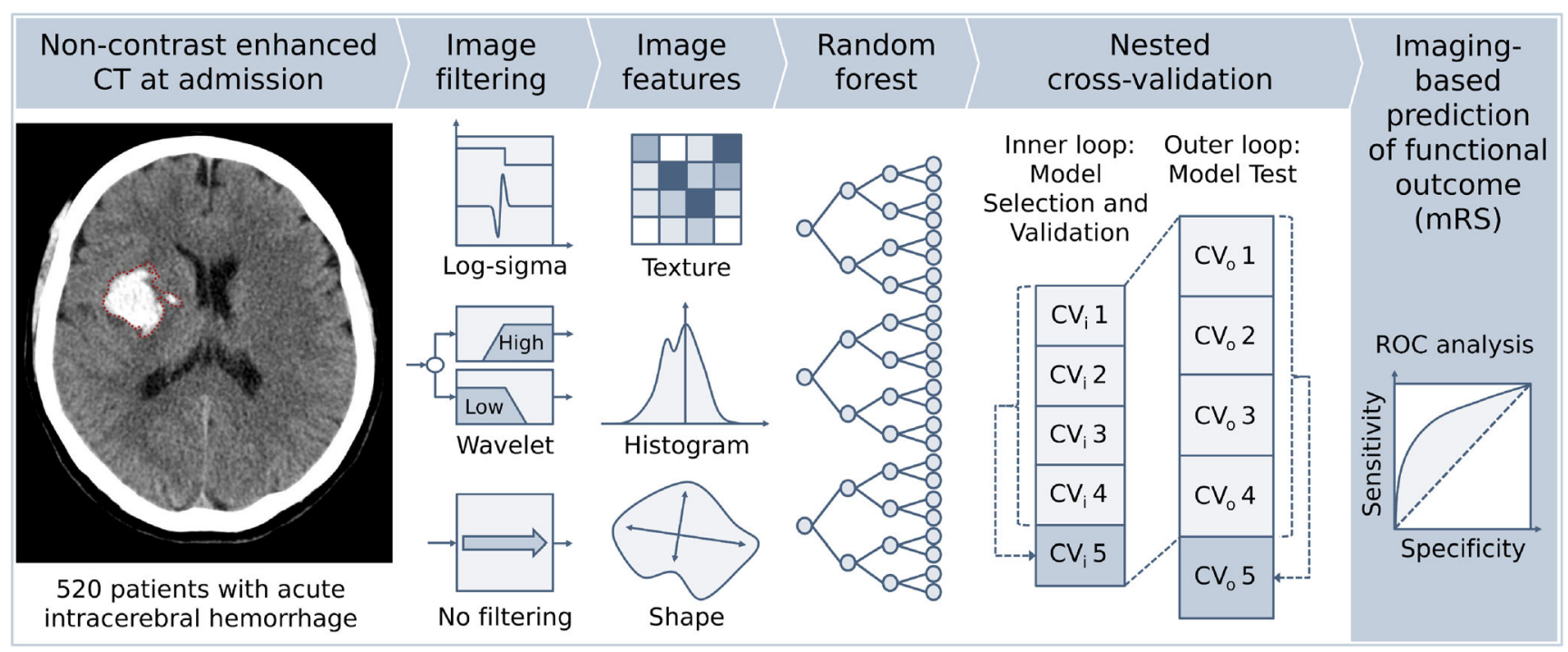

Fig. 1 Conceptual overview of the proposed machine learning approach for intracerebral hemorrhage outcome prediction showing the major processing steps: CT based image acquisition and segmentation, feature extraction $(n=1218)$, and statistical learning (random forest algorithm).

NECT, non-contrast-enhanced computed tomography; ICH, intracerebral hemorrhage; CT, computed tomography; mRS, modified Rankin Scale; $\mathrm{CV}$, cross-validation set with i: inner loop and o: outer loop

CI $[0.77 ; 0.82])$ for $\mathrm{mRS} \leq 2,0.80(95 \% \mathrm{CI}[0.78 ; 0.81])$ for $\mathrm{mRS} \leq 3$, and 0.79 (95\% CI $[0.77 ; 0.80])$ for $\mathrm{mRS} \leq 4$. Trained on survival prediction $(\mathrm{mRS} \leq 5)$, the classifier reached ROC AUCs of 0.80 (95\% CI [0.78; 0.82]) which was equivalent to results of the ICH Score with ROC AUC of 0.80 (95\% CI [0.79; 0.82]) (Fig. 2, Table 2). Exclusion of the parameter craniectomy yes/no had no effect on classification performance. Model selection and hyperparameter tuning within the nested cross-validation process resulted in the following median settings for $\mathrm{mRS} \leq 2, \leq 3, \leq 4$, and $\leq 5$, respectively (medians over cross-validation sets): Number of features considered: 25 , 100, 200, 100; number of trees: 750, 1000, 500, 1000; maximum depth of trees: 10 for all cut-off values; number of features considered for splitting $\left(\mathrm{m}_{\text {try }}\right)$, minimum number of samples to split an internal node, and minimum number of samples at leaf node: 1 for all cut-off values. Feature importance analyses of the mean top 100 predictors of all training data sets suggests that features with highest predictive power are mainly derived from wavelet $(43 \%)$ and log-sigma (30\%) filtered images. Unfiltered original images contributed $27 \%$ to total predictive power. Within feature classes, texture metrics dominated predictions (58\%) (Fig. 3). Predictive power of the 15 most important features demonstrates dominance of texture and shape features compared to first-order metrics (basic statistical measures of the grey level distribution). To also assess the predictive value of the ICH volume only, an additional ROC analysis was performed (supplementary Figure 1). ROC AUC for ICH volume as sole predictor was 0.72 with a Youden Index of 0.30 at $60 \%$ specificity and $70 \%$ sensitivity.

\section{Integration of ICH Score and Imaging-Based Outcome Prediction}

ICH Score metrics reached a ROC AUC of 0.80 (95\% CI $[0.79 ; 0.82])$, which was equivalent to the purely imagingbased classifier with ROC AUC of 0.80 (95\% CI [0.78; $0.82]$ ). If combined, the integrated model showed a significantly higher ROC AUC of 0.84 (95\% CI [0.83; 0.86], $P$ value $<0.05$ ). Sensitivities of the integrated model were significantly higher at Youden Index maximum cut-offs with $77 \%$ vs. $74 \%$ sensitivity at $76 \%$ specificity, $P$ value $<0.05$ (Fig. 2, Table 2).

\section{Discussion}

In this study, we developed an imaging-based ML model for predicting the functional outcome of ICH patients. The proposed approach employing quantitative image features derived from NECT scans provided high discriminatory accuracy between good and poor functional outcome of ICH patients at different mRS cut-off values. This study is based on a large multicenter and heterogeneous imaging dataset of 520 patients that was acquired in clinical routine over almost a decade. The proposed classification is solely based on high-end image features without a priori information about the location of the hemorrhage and without controlling for factors such as patient conditions, image acquisition parameters, or scanner type. Observed classification performance and model stability across all nested cross-validation runs suggest sufficient generalizability of our results.

It is a well-known paradigm that the ICH volume profoundly impacts functional clinical outcome. Initially derived 
a

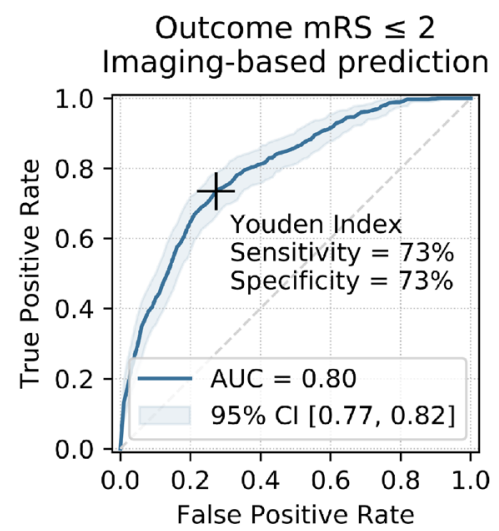

b

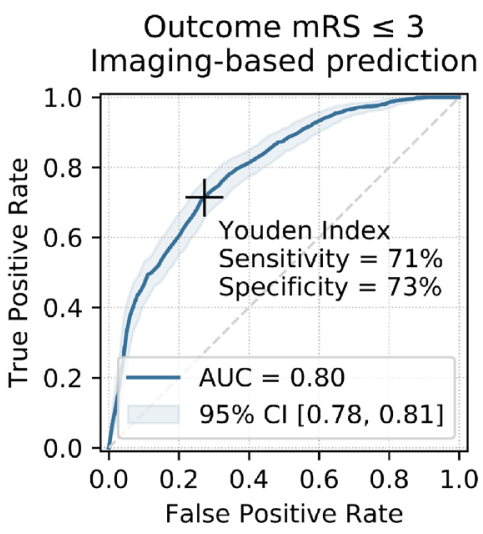

Survival $m R S<6$

Integrated prediction

Imaging-based + ICH score

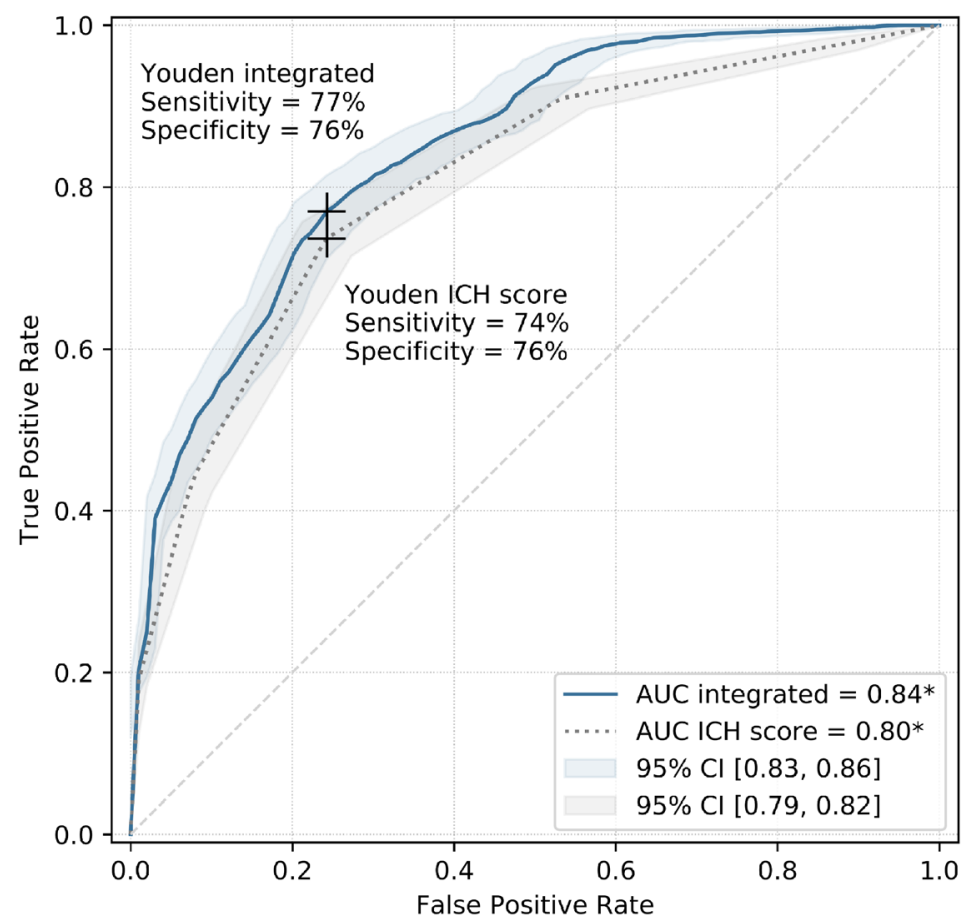

Outcome mRS $\leq 4$ Imaging-based prediction

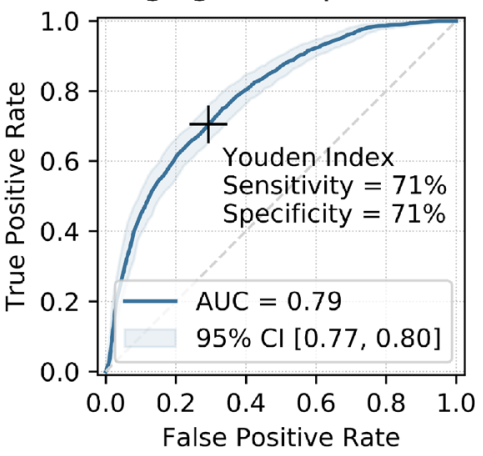

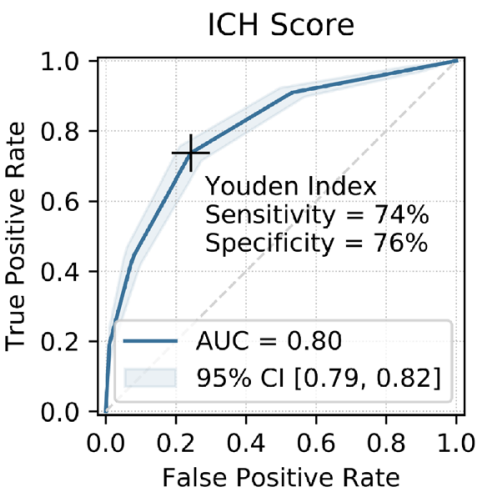

Imaging-based prediction

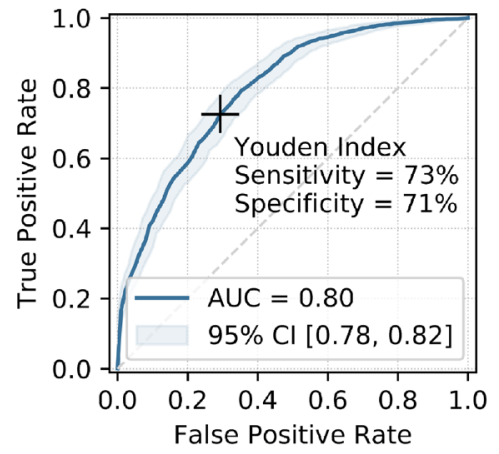

Fig. 2 Receiver-operating characteristics (ROC) curves for (a) functional outcome prediction of the proposed machine learning classifier based on quantitative image features and (b) prediction of survival using the ICH Score, the proposed machine learning classifier based on quantitative

by Broderick et al. to predict 30-day mortality after $\mathrm{ICH}$, the ICH volume has been later validated and included in the ICH Score $[3,18]$. In line with these findings, we have shown that ML-based outcome assessment using ICH volume as sole predictor already achieves ROC AUCs of $>0.70$ (supplementary Figure 1). Similarly, surrogate parameters of ICH volume such as maximum $2 \mathrm{D}$ diameter or minor axis length had comparatively high predictive importances in the imaging-based ML model. However, total contribution to predictive power of shape-based metrics in the comprehensive image features, and a classifier integrating ICH Score metrics and quantitative image features. AUC, area under the curve; CI, confidence interval; mRS, modified Rankin Scale

model was only $19 \%$ at ROC AUCs of 0.80 . It thus stands to reason that the ICH formation on NECT holds additional and relevant information which is not assessable by human eyes but can be evaluated by imaging-based ML algorithms. As so, analyses of the 100 most powerful features demonstrate the importance of second-order features (e.g., texture metrics) in comparison to first-order features. In contrast to first-order measures, second-order metrics also capture information regarding the spatial distribution of gray levels and are often difficult to evaluate by the human visual system. The 


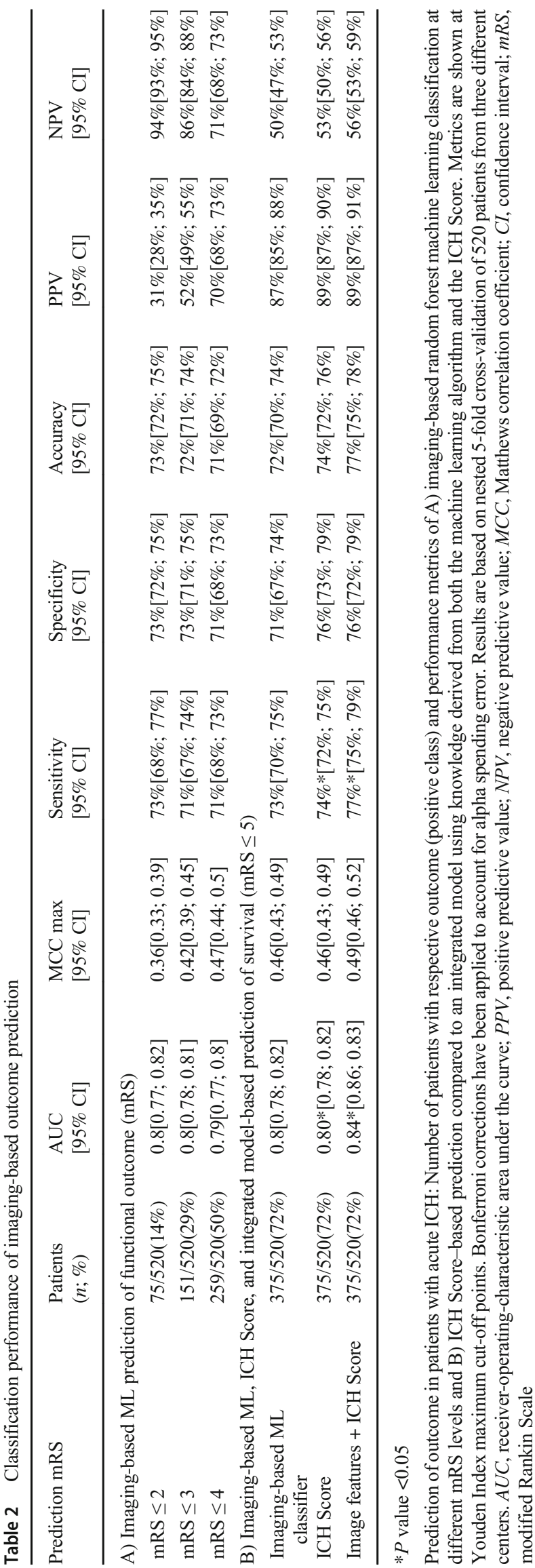

predictive value of second-order features is particularly apparent in the high predictive power of the gray level nonuniformity (Fig. 3). This specific finding could be related to the heterogenous appearance of hematomas that are still actively bleeding with evidence of spot sign or in those of patients with anticoagulation that are at risk for further expansion. It is equally conceivable that the gray level nonuniformity may differentiate areas of hyperacute ICH as the blend sign - with blending of a hypoattenuating area and a hyperattenuating region relative to the surrounding brain parenchyma - suggesting hematoma expansion and in reversal poor clinical outcome.

Hence, the proposed approach can be used as supportive tool to augment conventional image analysis and to improve prognostic decision for both radiologists and clinicians. As aspects of precision medicine are an emerging concept [30], combining the ICH Score with high-end imaging features may be useful in this respect. In line with this, the ICH Score seems to be limited in extension to critical care patients. In a prospective multicenter cohort study with patients presenting with spontaneous ICH and admitted to the intensive care unit (ICU), the ICH Score had only acceptable discriminatory power [31]. Although at this stage speculative and part of future studies, the proposed ML classifier may provide promising complementary results. In anticoagulation-associated $\mathrm{ICH}$, the ICH Score may not be as reliable $[21,32,33]$ and clinical outcomes in these patients likewise substantially often worse in comparison to patients without oral anticoagulation (OAC) $[34,35]$. Assuming that OAC therapy alters morphology and intensity of ICH, it is most likely that radiomic features are affected by OAC therapy. As we trained the ML model on acute $\mathrm{CT}$ images of both, patients receiving OAC and patients without $\mathrm{OAC}$, the information on OAC therapy is incorporated in the model through these differences in $\mathrm{ICH}$ imaging characteristics.

Since our quantitative imaging feature analysis performs equally in comparison to multidimensional scoring systems (e.g., ICH Score), the application of the proposed ML approach may be of value for randomized clinical trials. Challenges and opportunities to optimize clinical research and randomized trials in ICH are ongoing [36]. The ML approach could simplify trial procedures by performing an imaging-based prediction of functional outcome or early mortality. Simultaneously the multicenter approach of this study takes local variations in practice into account which are necessary to reflect upon a successful trial planning. Furthermore, this approach may also be of value for telemedicine and remote prediction of ICH outcome in regions lacking neuroradiological specialists. Taken together, the proposed method integrates the merits from quantitative radiomic features and ML algorithms and relates the employed predictors to wellknown imaging characteristics. 
Predictive power of top 15 quantitative image features

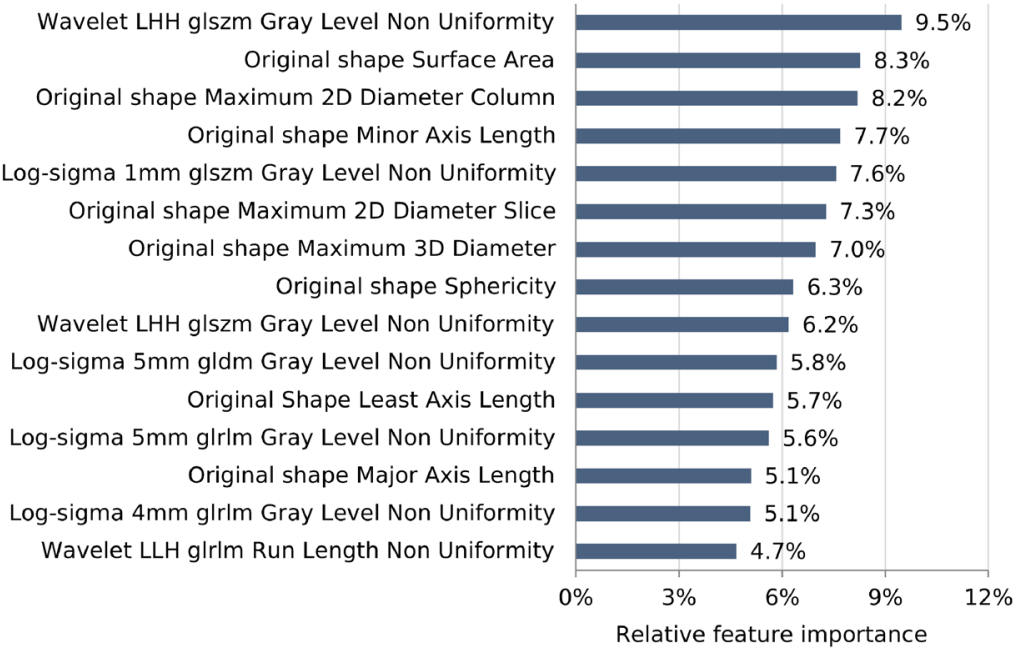

Feature class distribution

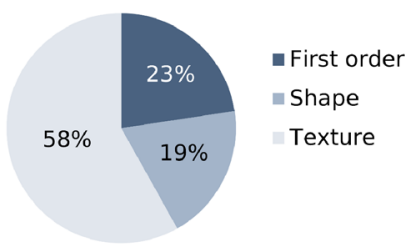

Applied filters

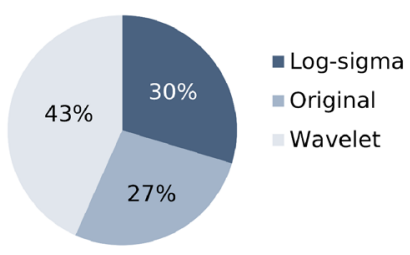

Fig. 3 Predictive value of quantitative image features. Bar charts show mean Gini impurity feature importance of all cross-validation training sets of the top- 15 high-end image features. Pie charts show distribution of feature classes and applied filters in utilized top- 100 predictors. First- order metrics: Basic statistical metrics of the voxel grey level distribution; glcm: gray level co-occurrence matrix; gldm: gray level dependence matrix; glrlm: gray level run length matrix; glszm: gray level size zone; $\mathrm{H}$ : high-pass wavelet decomposition; L: low-pass wavelet decomposition
Despite the promising results, several limitations deserve comment. Our study had general limitations typically associated with quantitative radiomics-based image analysis and classification [17, 37-39]. These limitations include differences in image acquisition settings (e.g., size of the field of view, gantry tilt) and under- or overfitting of machine learning algorithms. Bias of these factors was minimized through (a) employment of NECT scans that offer standardized HU metrics and (b) the application of random forest algorithms that are comparably stable with regard to overfitting. The risk of overfitting was further reduced by evaluating multiple different models in a nested cross-validation approach. Furthermore, we observed study-specific limitations: First, we included a limited number of patients in a retrospective analysis. An expansion of sample size in a prospective study design would certainly contribute to further improving generalizability of our results. However, observed model stability suggests sufficient robustness for evaluating feasibility and limitations of the proposed algorithm. The utilized dataset includes imaging data from 520 patients acquired over a relatively long period of almost a decade in three different centers. In such heterogeneous datasets, results of nested crossvalidation approaches serve as a valid indicator for confirming feasibility and performance of the proposed classifier in the underlying clinical setting. Due to standardized and calibrated quantitative imaging parameters and signal intensity processing of CT scanners, we assume neglectable bias on classifier performance in a generalized setting. Second, the manual definition of ROIs still implies a certain degree of observer dependence within the ML process. To minimize its influence, we employed consensus segmentations from two independent readers and applied a semi-automated delineation method that was shown to have a favorable inter- and intra-observer reliability and a high level of congruence with a fully automated delineation [20, 40]. Furthermore, it was found that radiomic features are relatively stable with regard to variations in segmentations [41, 42]. The lack of data on withdrawal and limitation of care are a further limitation [43]. Final limitation was the missing correlation with long-term data (e.g., mRS at 90 days and mortality) as it might offer additional information but was not available for this study [44].

\section{Conclusion}

Quantitative imaging features of acute NECT evaluated by ML algorithms provide a high discriminatory power in predicting functional outcome in patients with spontaneous ICH. Additional integration of the ICH Score increases predictive power of the ML classifier, hence providing promising complementary results. The findings support the potential of ML algorithms to augment conventional image analysis, improve prognostic decision, and simplify trial procedures. In the very near future, such ML techniques may play a pivotal role in determining optimized therapeutic regimes and predicting the prognosis for patients with ICH in an individualized manner.

Supplementary Information The online version contains supplementary material available at https://doi.org/10.1007/s12975-021-00891-8.

Acknowledgements Open Access funding enabled and organized by Projekt DEAL. 
Author Contribution - Substantial contributions to conception and design: JN, HK, JF, UH, GT, FQ

- Acquisition and analysis and interpretation of data: SE, CF, PS, LD, AM, GB, GS, TR, MB $\mathrm{UH}$

Drafting a significant portion of the manuscript or figures: JN, HK,

\section{Declarations}

Conflict of Interest - J. Fiehler: Consultant for Acandis, Boehringer Ingelheim, Codman, Microvention, Sequent, Stryker. Speaker for Bayer Healthcare, Bracco, Covidien/ev3, Penumbra, Philips, Siemens. Grants from Bundesministeriums für Wirtschaft und Energie (BMWi), Bundesministerium für Bildung und Forschung (BMBF), Deutsche Forschungsgemeinschaft (DFG), European Union (EU), Covidien, Stryker (THRILL study), Microvention (ERASER study), Philips.

- G. Thomalla has received personal fees from Acandis, Bayer, Boehringer Ingelheim, Bristol- Myers Squibb/Pfizer, Daichi Sankyo, Stryker, grants and personal fees from Bayer, grants from the German Research Foundation, Corona Foundation, German Innovation Fund.

- All other authors declare that they have no conflict of interest with a company whose product is used in the study or may be affected by its outcome. Please refer to the ICMJE Form for Disclosure of Potential Conflicts of Interest for further details.

Open Access This article is licensed under a Creative Commons Attribution 4.0 International License, which permits use, sharing, adaptation, distribution and reproduction in any medium or format, as long as you give appropriate credit to the original author(s) and the source, provide a link to the Creative Commons licence, and indicate if changes were made. The images or other third party material in this article are included in the article's Creative Commons licence, unless indicated otherwise in a credit line to the material. If material is not included in the article's Creative Commons licence and your intended use is not permitted by statutory regulation or exceeds the permitted use, you will need to obtain permission directly from the copyright holder. To view a copy of this licence, visit http://creativecommons.org/licenses/by/4.0/

\section{References}

1. Drury I, Whisnant JP, Garraway WM, Kissela B, Kleindorfer D, Moomaw CJ, et al. Primary intracerebral hemorrhage: impact of CT on incidence. Neurology. 1984;34:653-7.

2. Jakubovic R, Aviv RI. Intracerebral hemorrhage: toward physiological imaging of hemorrhage risk in acute and chronic bleeding. Front Neurol. 2012;3:86.

3. Broderick JP, Brott TG, Duldner JE, Tomsick T, Huster G. Volume of intracerebral hemorrhage. A powerful and easy-to-use predictor of 30-day mortality. Stroke. 1993;24:987-93.

4. Moullaali TJ, Wang X, Martin RH, Shipes VB, Robinson TG, Chalmers J, et al. Blood pressure control and clinical outcomes in acute intracerebral haemorrhage: a preplanned pooled analysis of individual participant data. Lancet Neurol. 2019;18:857-64.

5. Hemphill JC, Greenberg SM, Anderson CS, Becker K, Bendok BR, Cushman M, et al. Guidelines for the management of spontaneous intracerebral hemorrhage. Stroke. 2015;46:2032-60.

6. Selim M. Unmet needs and challenges in clinical research of intracerebral hemorrhage. Stroke. 2018;49:1299-307.

7. Gregório T, Pipa S, Cavaleiro P, Atanásio G, Albuquerque I, Chaves PC, et al. Prognostic models for intracerebral hemorrhage: systematic review and meta-analysis. BMC Med Res Methodol. 2018;18:145.

8. Heo J, Yoon JG, Park H, Kim YD, Nam HS, Heo JH. Machine learning-based model for prediction of outcomes in acute stroke. Stroke. 2019;50:1263-5.

9. Rubbert C, Patil KR, Beseoglu K, Mathys C, May R, Kaschner $\mathrm{MG}$, et al. Prediction of outcome after aneurysmal subarachnoid haemorrhage using data from patient admission. Eur Radiol. 2018;28:4949-58.

10. Rau C-S, Kuo P-J, Chien P-C, Huang C-Y, Hsieh H-Y, Hsieh C-H. Mortality prediction in patients with isolated moderate and severe traumatic brain injury using machine learning models. PLoS One. 2018;13:e0207192.

11. Arbabshirani MR, Fornwalt BK, Mongelluzzo GJ, Suever JD, Geise BD, Patel AA, et al. Advanced machine learning in action: identification of intracranial hemorrhage on computed tomography scans of the head with clinical workflow integration. npj Digit Med. 2018;1:9.

12. Scherer M, Cordes J, Younsi A, Sahin Y-A, Götz M, Möhlenbruch $\mathrm{M}$, et al. Development and validation of an automatic segmentation algorithm for quantification of intracerebral hemorrhage. Stroke. 2016;47:2776-82.

13. Wang H-L, Hsu W-Y, Lee M-H, Weng H-H, Chang S-W, Yang J$\mathrm{T}$, et al. Automatic machine-learning-based outcome prediction in patients with primary intracerebral hemorrhage. Front Neurol. 2019;10:910.

14. Morotti A, Boulouis G, Dowlatshahi D, Li Q, Barras CD, Delcourt C, et al. Standards for detecting, interpreting, and reporting noncontrast computed tomographic markers of intracerebral hemorrhage expansion. Ann Neurol. 2019;86:480-92.

15. Barras CD, Tress BM, Christensen S, Collins M, Desmond PM, Skolnick BE, et al. Quantitative CT densitometry for predicting intracerebral hemorrhage growth. Am J Neuroradiol. 2013;34: 1139-44.

16. Soun JE, Montes D, Yu F, Morotti A, Qureshi AI, Barnaure I, et al. Spot Sign in Secondary Intraventricular hemorrhage predicts rarly neurological decline. Clin Neuroradiol. 2019;1-8 [Online ahead of print].

17. Kniep HC, Madesta F, Schneider T, Hanning U, Schönfeld MH, Schön G, et al. Radiomics of brain MRI: utility in prediction of metastatic tumor type. Radiology. 2019;290:479-87.

18. Hemphill JC, Bonovich DC, Besmertis L, Manley GT, Johnston SC. The ICH score: a simple, reliable grading scale for intracerebral hemorrhage. Stroke. 2001;32:891-7.

19. Volbers B, Staykov D, Wagner I, Dörfler A, Saake M, Schwab S, et al. Semi-automatic volumetric assessment of perihemorrhagic edema with computed tomography. Eur J Neurol. 2011;18:1323-8.

20. Urday S, Beslow LA, Goldstein DW, Vashkevich A, Ayres AM, Battey TWK, et al. Measurement of perihematomal edema in intracerebral hemorrhage. Stroke. 2015;46:1116-9.

21. Houben R, Schreuder FHBM, Bekelaar KJ, Claessens D, van Oostenbrugge RJ, Staals J. Predicting prognosis of intracerebral hemorrhage (ICH): performance of ICH score is not improved by adding oral anticoagulant use. Front Neurol. 2018;9:100.

22. Pedregosa F, Varoquaux G, Gramfort A, Michel V, Thirion B, Grisel O, et al. Scikit-learn: machine learning in python. J Mach Learn Res. 2011;12:2825-30.

23. Breiman L. Mach Learn 2001;45:5-32.

24. Applications. Cha Zhang $\bullet$ Yunqian Ma Editors ensemble machine learning. [cited 2019 Aug 26];Available from: www.springer.com

25. Louppe G, Wehenkel L, Sutera A GP. Understanding variable importances in forests of randomized trees. Proc. 26th Int. Conf. Neural Inf. Process. Syst. 2013;1:431-439.

26. Limkin EJ, Sun R, Dercle L, Zacharaki EI, Robert C, Reuzé S, et al. Promises and challenges for the implementation of computational 
medical imaging (radiomics) in oncology. Ann Oncol. 2017;28: 1191-206.

27. Robin X, Turck N, Hainard A, Tiberti N, Lisacek F, Sanchez J-C, et al. pROC: an open-source package for R and S+ to analyze and compare ROC curves. BMC Bioinformatics. 2011;12:77.

28. Matthews BW. Comparison of the predicted and observed secondary structure of T4 phage lysozyme. BBA - Protein Struct. 1975;405:442-51.

29. Powers DMW, Evaluation: from precision, recall and F-measure to ROC, informedness, markedness \& correlation. 2011;2:37-63.

30. Hamburg MA, Collins FS. The path to personalized medicine. $\mathrm{N}$ Engl J Med. 2010;363:301-4.

31. Rodríguez-Fernández S, Castillo-Lorente E, Guerrero-Lopez F, Rodríguez-Rubio D, Aguilar-Alonso E, Lafuente-Baraza J, et al. Validation of the ICH score in patients with spontaneous intracerebral haemorrhage admitted to the intensive care unit in Southern Spain. BMJ Open. 2018;8:e21719.

32. Katsanos AH, Krogias C, Lioutas VA, Goyal N, Zand R, Sharma VK, et al. The prognostic utility of ICH-score in anticoagulant related intracerebral hemorrhage. J Neurol Sci. 2020;409:116628.

33. Fakiri MO, Uyttenboogaart M, Houben R, van Oostenbrugge RJ, Staals J, Luijckx GJ. Reliability of the intracerebral hemorrhage score for predicting outcome in patients with intracerebral hemorrhage using oral anticoagulants. Eur J Neurol. 2020;27:2006-13.

34. Morotti A, Goldstein JN. Anticoagulant-associated intracerebral hemorrhage. Brain Hemorrhages. 2020;1:89-94.

35. Boulouis G, Morotti A, Pasi M, Goldstein JN, Gurol ME, Charidimou A. Outcome of intracerebral haemorrhage related to non-Vitamin $\mathrm{K}$ antagonists oral anticoagulants versus Vitamin $\mathrm{K}$ antagonists: a comprehensive systematic review and meta-analysis. J Neurol Neurosurg Psychiatry. 2018;89:263-70.
36. Selim M, Hanley D, Steiner T, Christensen HK, Lafuente J, Rodriguez D, et al. Recommendations for clinical trials in ICH. Stroke. 2020;51:1333-8.

37. Gillies RJ, Kinahan PE, Hricak H. Radiomics: images are more than pictures, They Are Data. Radiology. 2016;278:563-77.

38. Aerts HJWL. The potential of radiomic-based phenotyping in precision medicine. JAMA Oncol. 2016;2:1636-42.

39. Lambin P, Leijenaar RTH, Deist TM, Peerlings J, de Jong EEC, van Timmeren J, et al. Radiomics: the bridge between medical imaging and personalized medicine. Nat Rev Clin Oncol. 2017;14:749-62.

40. Ironside N, Chen CJ, Mutasa S, Sim JL, Marfatia S, Roh D, et al. Fully automated segmentation algorithm for hematoma volumetric analysis in spontaneous intracerebral hemorrhage. Stroke. 2019;50: 3416-23.

41. Parmar C, Rios Velazquez E, Leijenaar R, Jermoumi M, Carvalho $\mathrm{S}$, Mak RH, et al. Robust radiomics feature quantification using semiautomatic volumetric segmentation. PLoS One. 2014;9: e102107.

42. Yip SSF, Aerts HJWL. Applications and limitations of radiomics. Phys Med Biol. 2016;61:R150-66.

43. Zahuranec DB, Brown DL, Lisabeth LD, Gonzales NR, Longwell PJ, Smith MA, et al. Early care limitations independently predict mortality after intracerebral hemorrhage. Neurology. 2007;68: 1651-7.

44. Selim M, Hanley D, Steiner T, Christensen HK, Lafuente J, Rodriguez D, et al. Recommendations for clinical trials in $\mathrm{ICH}$ : the second hemorrhagic stroke academia industry roundtable. Stroke. 2020;51:1333-8.

Publisher's Note Springer Nature remains neutral with regard to jurisdictional claims in published maps and institutional affiliations. 